\title{
Bone Marrow Tracer Uptake
}

National Cancer Institute

\section{Source}

National Cancer Institute. Bone Marrow Tracer Uptake. NCI Thesaurus. Code C147490.

A visually assessed combination of extent and intensity of tracer uptake within the bone marrow. 